\title{
A line-up of new therapies and expanded combinations
}

Neil Osterweil and Patrice Wendling report from the 2015 annual meeting of the American Society of Hematology in Orlando, Florida

\section{First-line ibrutinib beats standard chemo for CLL, SLL in older patients \\ Key clinical point First-line ibrutinib significantly extends} survival in older patients with untreated CLL or SLL, compared with chlorambucil chemotherapy. Major finding Median PFS was not reached with ibrutinib vs 19 months with chlorambucil $(H R, 0.16 ; P<.001)$. Data source Prospective, phase 3 study of 269 patients aged 65 years or older with treatmentnaive CLL or SLL. Disclosures Pharmacyclics, which is jointly developing ibrutinib with Janssen Biotech, sponsored the study. Dr Tedeschi reported having nothing to disclose. Several coauthors reported relationships with Pharmacyclics and Janssen.

Monotherapy with ibrutinib prolonged survival longer than did standard chemotherapy using chlorambucil in the front-line treatment of older patients with chronic lymphocytic leukemia (CLL) or small lymphocytic lymphoma (SLL) in the phase 3 RESONATE-2 study.

The developers of the drug had announced last summer that ibrutinib, an orally bioavailable, small-molecule inhibitor of Bruton's tyrosine kinase, had achieved its primary and secondary endpoints. But the first full look at the data at the meeting showed ibrutinib reduced the risk of progression or death by $84 \%$ by independent review, compared with chlorambucil, which has been a standard first-line therapy in older CLL patients.

With a median follow-up of 18.4 months, median progression-free survival (PFS) had not been reached with ibrutinib, compared with 19 months with chlorambucil (hazard ratio [HR], 0.16; $P<.001$ ).

By investigator assessment, ibrutinib reduced the risk of progression by $91 \%$, with an 18 -month PFS rate of $94 \%$, compared with $45 \%$ with chlorambucil (HR, 0.09; $P<$ .001).

The PFS benefit with ibrutinib was consistent regardless of patient age, Rai stage, ECOG (Eastern Cooperative Oncology Group) status, bulky disease, high-risk markers such as chromosome 11q deletion and unmutated immunoglobulin heavy chain variable mutation status, said study author Dr Alessandra Tedeschi of Hospital Niguarda Cà Granda, Milan. In addition, ibrutinib led to an $84 \%$ reduction in the risk of death, compared with chlorambucil (HR, $0.16 ; P=.001)$. The 24 -month overall survival rate was $98 \%$ with ibrutinib, compared with $85 \%$ with chlorambucil.

Single-agent ibrutinib was approved in 2014 for patients with CLL who had received at least 1 prior therapy and for all patients with the deleterious $17 \mathrm{p}$ deletion on the basis of the phase 3 RESONATE trial in relapsed or refractory CLL. Three-year follow-up in the phase 2 PCYC-1102 study signaled a benefit with ibrutinib in treatment-naive CLL, showing an overall response rate of 84\%, 30-month PFS of $96 \%$, and overall survival rate of $97 \%$ in a subset of 31 patients at least 65 years old (Blood 2015;125:2497-2506).

"The phase 3 RESONATE-2 trial confirms the efficacy of ibrutinib in treatment-naive CLL patients, leading to a 91\% reduction in risk of progression and $84 \%$ reduction in risk of death compared with chlorambucil," Dr Tedeschi said.

In all, 269 patients, median age of 73 years, were evenly randomized to once-daily ibrutinib $420 \mathrm{mg}$ until progression or unacceptable toxicity or chlorambucil $0.5 \mathrm{mg} / \mathrm{kg}$ (to a maximum of $0.8 \mathrm{mg} / \mathrm{kg}$ ) on days 1 and 15 of a 28 -day cycle for up to 12 cycles. Patients with the deleterious $17 \mathrm{p}$ deletion were excluded because single-agent chlorambucil is not effective in that population.

Ibrutinib significantly improved bone marrow function, as reflected by a sustained increase in hemoglobin and platelets. "This is very important in this category of elderly patients, in whom bone marrow failure is the most common cause of morbidity," Dr Tedeschi said.

There were 3 deaths on the ibrutinib arm and 17 on the chlorambucil arm.

The majority of patients (87\%) in this older population with frequent comorbidities was able to continue on oral, once-daily ibrutinib with a median of 1.5 years of followup, she said.

The most common adverse events on ibrutinib were grade 1 diarrhea, fatigue, cough, and nausea that did not result in treatment discontinuation. On the chlorambucil arm, fatigue, nausea, vomiting, and cytopenias occurred more frequently than with ibrutinib. Grade 3 maculopapular rash occurred in $3 \%$ with ibrutinib and $2 \%$ with chlorambucil, she said.

Ibrutinib was associated with higher and not insignificant rates of atrial fibrillation and major hemorrhage 
compared with chlorambucil, said Dr Brian T Hill of the Taussig Cancer Institute at the Cleveland Clinic, who was not involved in the study. In an interview, Dr Hill also questioned the relevance today of chlorambucil monotherapy as the comparator arm in RESONATE-2.

\section{- Patrice Wendling}

\section{All-oral regimen extends multiple myeloma progression-free survival \\ Key clinical point Ixazomib added to lenalidomide and} dexamethasone is an effective all-oral drug regimen in relapsed/refractory multiple myeloma. Major finding The combination was associated with a $35 \%$ improvement in PFS, compared with len-dex and placebo. Data source Randomized placebo-controlled trial in 722 patients with relapsed/refractory multiple myeloma treated with 1 or 3 prior lines of therapy. Disclosures Millennium Pharmaceuticals sponsored the study. Dr Moreau disclosed receiving honoraria from Millennium.

The first all-oral drug combination for treatment of relapsed/ refractory multiple myeloma significantly extended progression-free survival (PFS), based on the first of 3 planned interim analyses from a phase 3 trial.

The combination of the recently approved oral proteasome inhibitor ixazomib with lenalidomide and dexamethasone (len-dex) was associated with a $35 \%$ improvement in PFS, compared with len-dex and placebo, Dr Philippe Moreau from Université Hospital of Nantes (France), Hôtel-Dieu, reported at the meeting.

The ixazomib combination also was associated with a significantly better median time to progression compared with the placebo group (21.4 vs 15.7 months; $P=.007$ ). However, the time to response and duration of response were not significantly different for the 2 groups in the placebo-controlled, randomized study of 722 patients.

"Ixazomib, when combined with len-dex for patients with relapsed and/or refractory multiple myeloma, was associated with a significant and meaningful improvement in progression-free survival, significantly improved time to progression, and confirmed response rates," Dr Moreau said.

Dr Moreau presented results of the phase 3 Tourmaline study, which compared len-dex plus weekly ixazomib or len-dex with weekly placebo in adults who have relapsed/ refractory multiple myeloma following 1 to 3 prior lines of therapy and were not refractory to either prior lenalidomide or proteasome inhibitor-based therapy.

The study met its primary endpoint of a PFS advantage for the addition of ixazomib. The median PFS for ixazomib plus len-dex was 20.6 months, compared with 14.7 months for len-dex plus placebo. The hazard ratio for PFS with ixazomib-containing combination was $0.742(P=.012)$.

The confirmed overall response rate (partial response or greater) was $78 \%$ with ixazomib and $71 \%$ with placebo $(P$
$=.035)$. The respective very good partial response or better rates were $48 \%$ and $39 \%(P=.014)$.

A total of 722 patients (overall median age, 66 years; range, 30-91) were randomized to either ixazomib $4 \mathrm{mg}$ or placebo weekly on days $1,8,15$, and 22 of each 28 -day cycle, with oral lenalidomide $25 \mathrm{mg}$ on days 1 through 21 (with dose reductions in patients with renal impairment at the investigator's discretion) and oral dexamethasone 40 $\operatorname{mg}$ on days $1,8,15$, and 22 .

Patients were stratified at randomization by the number of prior therapies $(1,2$, or 3$)$, previous proteasome-inhibitor exposure, and by International Staging System for multiple myeloma stage I or II versus III. Cycles were repeated until disease progression or until a patient experienced unacceptable toxicity. In all, $70 \%$ had received a prior proteasome inhibitor, $88 \%$ had International Staging System stage I or II disease, and 59\% had received 1 prior line of therapy. Dr Moreau noted that the responses were rapid and durable, with a median time to response of 1.1 months for ixazomib and 1.9 months for placebo, and a median duration of response of 20.5 months and 15 months, respectively. Neither difference was significant.

The incidence of grade 3 or greater adverse events was $68 \%$ for patients on ixazomib and $61 \%$ with placebo. A higher incidence of thrombocytopenia with ixazomib than with placebo (19\% vs 9\%) primarily accounted for the difference. Dr Moreau noted that peripheral neuropathy, a common problem with intravenous proteasome inhibitors, was low with ixazomib, and patients did not report significant changes in their quality of life.

— Neil Osterweil

\section{Upfront idelalisib carries high risk for acute liver toxicity \\ Key clinical point Idelalisib in the first-line setting is as-} sociated with significant risk of hepatotoxicity, with a peak incidence at about 28 days of therapy. Major finding More than half $(52 \%)$ of patients with newly diagnosed CLL had grade 3 or 4 hepatotoxicity with idelalisib monotherapy.

Data source Ongoing phase 2 clinical trial with data on 24 patients. Disclosures The Dana-Farber Cancer Institute in collaboration with Gilead Sciences and GlaxoSmithKline sponsored the study. Dr Lampson and colleagues declared no relevant conflicts of interest.

Idelalisib given as first-line therapy for patients with chronic lymphocytic leukemia (CLL) carries a high risk of early fulminant hepatotoxicity requiring drug interruption and steroids, investigators reported at the meeting.

Among 24 patients who received idelalisib monotherapy in a phase 2 trial of a combination of idelalisib followed by idelalisib concurrent with ofatumumab as first-line therapy for CLL, 12 patients developed acute hepatotoxicity, marked by rapidly soaring levels of transaminase within about 28 days of starting therapy. An additional 4 patients 
developed hepatotoxicity at around 130 days, noted Dr Benjamin L Lampson, a clinical fellow in medicine at the Dana-Farber Cancer Institute in Boston.

"Multiple lines of evidence suggest that this early hepatotoxicity is immune mediated. The proportion of regulatory $\mathrm{T}$ cells in the peripheral blood decrease on idelalisib therapy, providing a possible explanation for the development of early hepatotoxicity," he said.

The toxicities occur more frequently in younger and less heavily pretreated patients, and are likely owing to on-target, immune-mediated effects, he noted.

Dr Lampson presented data on the first 24 patients in an ongoing phase 2 trial. Patients with previously untreated CLL receive idelalisib $150 \mathrm{mg}$ twice daily for 56 days to mobilize neoplastic B cells from the peripheral lymphoid tissues and into the bloodstream. After the monotherapy phase, patients are given ofatumumab in an attempt to clear the disease from peripheral blood. "This dosing strategy is slightly different than what has previously been used in trials combining these particular drugs. Specifically, previously reported trials have started these agents simultaneously without a lead-in period of monotherapy," Dr Lampson explained.

When the lead-in phase is completed, patients receive idelalisib plus ofatumumab infusions once weekly for 8 weeks, followed by once-monthly infusions for 4 months. Patients then continue on idelalisib indefinitely. The primary endpoint is the overall response rate assessed 2 months after the completion of the combination therapy.

For the first 24 patients treated as of November 9, 2015, the median time on therapy was 7.7 months and median follow-up was 14.7 months. The median patient age was 67.4 years (range, 57.6-84.9). CLL genetics showed that 13 patients had unmutated immunoglobulin heavy chain variable region disease, 4 had the 17p deletion and TP53 mutation, 1 had deletion 11q, and 13 had deletion 13q; some patients had more than 1 mutation.

"We began to notice after enrolling just a few subjects on the trial that severe hepatotoxicity was occurring shortly after initiating idelalisib," Dr Lampson said. He presented the case of a 58-year-old man who was in the idelalisib monotherapy phase of the study. The patient developed grade 3 hepatotoxicity 28 days after starting the drug, despite having a normal liver function test just 1 day earlier.

The drug was stopped, but his liver function tests continued to rise, suggesting a self-perpetuating or self-sustaining process. On day 32 , the patient was admitted to the hospital, and on day 33 he was started on steroids, based on the hypothesis that the hepatotoxicity might have been immune mediated. Two days after initiation of steroids, his liver function tests continued to rise, whereupon he was started on mycophenolate mofetil.

"With these 2 forms of immunosuppression, the [liver function tests] did eventually normalize, although the ste- roids and mycophenolate had to be tapered over a period of many weeks. And this patient was not the only patient to experience toxicity; in fact, hepatotoxicity was frequent and often severe," he said.

At the time of maximum incidence, week 4, the percentage of patients with any hepatotoxicity was $46 \%$, with $13 \%$ at grade 4 and $21 \%$ at grade 3 .

"The median time to initial development of hepatotoxicity is 28 days. This suggests that the mechanism of hepatotoxicity is not immediate, but takes time to develop, consistent with an adaptive immune response. Furthermore, hepatotoxicity is typically occurring before the first dose of ofatumumab is occurring at week 8 , suggesting idelalisib alone is the cause of the hepatotoxicity," Dr Lampson said. - Neil Osterweil

\section{Idelalisib plus standard therapy boosts survival in relapsed CLL \\ Key clinical point Adding the PI3K inhibitor idelalisib to} bendamustine and rituximab significantly improved survival of patients with relapsed/refractory CLL. Major finding Median PFS was 23.1 months for patients treated with idelalisib, bendamustine, and rituximab, compared with 11.1 months for bendamustine and rituximab plus placebo. Data source Randomized, controlled trial in 416 patients with relapsed/ refractory CLL. The trial was halted early for superior efficacy with idelalisib. Disclosures Gilead Sciences funded the study. Dr Zelenetz disclosed receiving research funding from Gilead and discussing off-label use of idelalisib for relapsed/ refractory

Adding the PI3K inhibitor idelalisib to a standard regimen of bendamustine and rituximab significantly reduced the risk of both disease progression and death for patients with relapsed and/or refractory chronic lymphocytic leukemia (CLL), results of a phase 3 randomized trial showed.

At a median follow-up of 12 months, the primary endpoint of median progression-free survival (PFS) was 23.1 months for patients treated with idelalisib, bendamustine, and rituximab (idel+BR), compared with 11.1 months for bendamustine and rituximab (BR) plus a placebo, reported Dr Andrew Zelenetz of Memorial Sloan Kettering Cancer Center, New York.

"Median overall survival was not reached in either arm. However, there was a significant improvement in overall survival, with a $45 \%$ reduction in the risk of death [with idel+BR]," he said at the meeting. The trial was stopped early after a data review at the first planned interim analysis showed significant superiority for the 3-drug combination.

The results were consistent across subgroups, including patients with high-risk features such as deletion $17 \mathrm{p}$ and mutated TP53 (del[17p]/TP53), unmutated immunoglobulin heavy chain variable region, and treatment-refractory disease.

The rationale behind adding idelalisib, an inhibitor of the phosphatidylinositol-3 kinase (PI3K), is that signaling via 
the PI3K pathway is hyperactive and can be targeted, Dr Zelenetz explained.

Study 115 was a phase 3 trial with accrual from June 2012 through August 2015. Investigators enrolled 416 patients with relapsed/refractory CLL and randomly assigned them to receive BR for six 28-day cycles of bendamustine (70 $\mathrm{mg} / \mathrm{m}^{2}$ on days 1 and 2 of each cycle) and rituximab ( 375 $\mathrm{mg} / \mathrm{m}^{2}$ for cycle 1 , and $500 \mathrm{mg} / \mathrm{m}^{2}$ for cycles 2 through 6 ), plus either idelalisib $150 \mathrm{mg}$ bid or placebo, each administered continuously until disease progression, intolerable toxicity, withdrawal of consent, or death.

The patients were stratified by mutational and disease status (refractory defined as CLL progression less than 6 months from completion of prior therapy, or relapsed CLL progression 6 months or more from completion of prior therapy.

The trial was halted early after the first planned interim analysis, which was conducted after $75 \%$ of the total number of 260 planned events of CLL progression or death from any cause had occurred. The data cut-off was June 15, 2015.

The intention-to-treat analysis included 207 patients assigned to idelalisib and 209 assigned to placebo. Threequarters of the patients (76\%) were men. In all, $46 \%$ of patients had Rai stage III/IV disease. The median time since the completion of the last therapy was 16 months. The proportions of patients with high-risk features included $\operatorname{del}(17 \mathrm{p}) / \mathrm{p} 53$ mutation in $32.9 \%$, unmutated $\mathrm{IgHV}$ in $83.2 \%$, and treatment-refractory disease in $29.8 \%$.

As noted, the median PFS with idelalisib at a median follow-up of 12 months was 23.1 months, compared with 11.1 months for placebo. That translated into a hazard ratio of $0.33(P<.0001)$.

Among patients with neither del(17p) nor TP53 mutations, the $\mathrm{HR}$ for progression was 0.22 . Among patients with either $\operatorname{del}(17 \mathrm{p})$ or a TP53 mutation, the HR was 0.50 (95\% confidence intervals show statistical significance for both).

Overall response rates were $68 \%$ among patients who received idelalisib, and $45 \%$ for those who received placebo. There were 5 complete responses $(2 \%)$ in the idelalisib group, and none in the placebo group.

The idelalisib group also had a higher proportion of patients with a greater than $50 \%$ reduction in involved lymph nodes (96\% vs 61\%), and had better organomegaly responses (spleen and liver) and hematologic responses (hemoglobin, neutrophils, and platelets).

Grade 3 or greater adverse events occurred in 93\% of patients on idelalisib, compared with $76 \%$ of those on placebo. The proportion of patients with any serious adverse event was $66 \%$ vs $44 \%$, respectively.

Adverse events leading to drug dose reduction were seen in $11 \%$ of idelalisib-treated patients, compared with $6 \%$ of placebo controls, and therapy was discontinued in $26 \%$ vs $13 \%$, respectively.

Ten patients in the idelalisib arm and seven in the placebo arm died during the study.
Adverse events that occurred more commonly with idelalisib included neutropenia, pyrexia, diarrhea, febrile neutropenia, pneumonia, rash, and elevated liver enzymes.

\section{- Neil Osterweil}

\section{Chemo quadruples risk for myeloid cancers \\ Key clinical point This study quantifies the risks for} treatment-related myeloid cancers after chemotherapy. Major

finding Chemotherapy is associated with a fourfold risk for treatment-related AML/MDS, compared with the general population. Data source Retrospective review of data on 746,007 adults treated with chemotherapy for a first primary malignancy. Disclosures The National Cancer Institute supported the study. Dr Morton reported having no conflicts of interest to disclose.

Patients who undergo cytotoxic chemotherapy, even in the modern era, are at increased risk for developing myeloid neoplasms, based on data from a cohort of nearly 750,000 adults who were initially treated with chemotherapy and survived at least 1 year after diagnosis.

In the cohort, the standardized incidence ratio (SIR) for treatment-related acute myeloid leukemia (tAML) or myelodysplastic syndrome (MDS) was 4 times greater than would be expected in the general population, reported Lindsay M Morton, $\mathrm{PhD}$, of the division of cancer epidemiology and genetics at the National Cancer Institute in Bethesda, Maryland. "We now demonstrate that there are elevated risks for treatmentrelated leukemia after treatment for a broad spectrum of first primary malignancies that are generally consistent with what we know about changing treatment practices," she said at meeting.

Dr Morton emphasized that there has been a notable increase in the number of cancer survivors in the United States, to the current 14 million, and that number is expected to rise to more than 18 million in the next few years. "[That] means that the long-term health of this population is of great clinical as well as public health importance," she said.

The link between cytotoxic chemotherapy and leukemia risk has been known since the 1960s, with certain classes of agents carrying higher risks than others, including platinum-containing compounds, alkylating agents (for example, cyclophosphamide, melphalan, chlorambucil), topoisomerase II inhibitors (eg, doxorubicin, daunorubicin, epirubicin), and antimetabolites (eg, 5-fluorauracil, capecitabine, gemcitabine).

Treatment-related leukemias are associated with higher doses of these agents, and the trend in contemporary medicine is to use more of these agents upfront for the treatment of primary malignancies. Yet estimates of the risk of tAML, MDS, or other malignancies have been hard to come by because of the relative rarity of cases and the sporadic reports in the literature, Dr Morton said. 
The investigators previously reported that risk for tAML and other myeloid neoplasms changed over time, and showed that since the 1990s there was an uptick in risk for patients treated with chemotherapy for cancers of bone, joints, and endometrium, and since 2000 for patients treated with chemotherapy for cancers of the esophagus, cervix and prostate.

For example, risks for tAML were higher in the 1970s for patients with ovarian cancer treated with melphalan, a highly leukemogenic agent, but dropped somewhat with the switch to platinum-based agents. Similarly, women with breast cancer had a comparatively high risk with the use of melphalan, a decline in risk with the introduction of cyclophosphamide, and then an increase with the addition of anthracyclines and dose-dense regimens.

To get a better idea of the magnitude of risk in the modern era, Dr Morton and colleagues sifted through Surveillance, Epidemiology, and End Results data to identify a cohort of 746,007 adults who were initially treated with chemotherapy and survived for at least 1 year following a diagnosis with a first primary malignancy during 2000-2012. They calculated SIRs based on variables that included age, race, sex, malignancy type, and treatment period.

The investigators looked at 4 categories of myeloid neoplasms as defined by World Health Organization criteria: AML/MDS, chronic myeloid leukemia, myeloproliferative neoplasms negative for BCR-ABL (Philadelphia-negative), and chronic myelomonocytic leukemia (CMML). They found that 2,071 patients developed treatment-related AML/MDS, translating into a fourfold incidence compared with the general population (SIR, 4.1; 95\% CI, 3.94.2), and that 106 were diagnosed with CMML.
They also identified novel risk for tAML/MDS after chemotherapy by malignancy.

The investigators found that breast cancer, non-Hodgkin lymphoma, and lung cancer were most commonly associated with tAML/MDS (SIRs 4.1, 7.3, and 4.1, respectively, all significant). In addition, although the overall numbers of cases were small, the investigators noted "strikingly elevated" risks for cancers of bone (SIR, 35.1; 95\% CI, 16.964.6), testes (SIR, 15.6; 95\% CI, 9.2-24.6), and soft tissue (SIR, 12.6; 95\% CI, 7.7-19.4),

Risk for tAML/MD was more modestly elevated for cancers of the brain, ovaries, endometrium, cervix, and prostate, and for Hodgkin lymphoma, chronic lymphocytic leukemia, and multiple myeloma.

Adding radiotherapy to chemotherapy for cancers of the breast, lung, and stomach cancers showed a trend toward heightened tAML/MDS risk, but this was not significant.

An elevated risk for CMML was also seen after chemotherapy for lung cancer (SIR, 2.5; 95\% CI, 1.3-4.4), breast cancer (SIR, 1.8; 95\% CI, 1.3-2.5), and non-Hodgkin lymphoma (SIR, 2.1; 95\% CI, 1.2-3.4). There was elevated risk for CMML following chemotherapy for breast cancer (SIR, 3.0; 95\% CI, 1.7-5.0) and non-Hodgkin lymphoma (SIR, 4.2; 95\% CI, 2.4-6.9).

There were no increased risks for other myeloproliferative neoplasms after chemotherapy for any first primary cancer, however. "This reminds us that with new uses of standard agents and introduction of new agents, it's critical to carefully weigh the risks and benefits of systemic therapy," Dr Morton said.

The investigators plan to quantify risks associated with specific drugs and doses, she added.

- Neil Osterweil 